\title{
METAPARADIGMA Y TEORIZACIÓN ACTUAL E INNOVADORA DE LAS TEORÍAS Y MODELOS DE ENFERMERÍA
}

\section{METAPARADIGM AND INNOVATIVE THEORIZATION ON NURSING THEORIES AND MODELS.}

\author{
Morales Valdivia, Estela \\ Rubio Contreras, Ana Ma \\ Ramírez Durán, Ma del Valle
}

Cursando Máster Oficial en Ciencias de la Enfermería. Universidad de Huelva

E-mail: estelamova@gmail.com

\section{RESUMEN}

Título: Metaparadigma y teorización actual e innovadora de las teorías y modelos de Enfermería.

\section{Objetivos:}

- Crear un metaparadigma compendio de las ideas esenciales y más actuales de las teóricas Orem, Roy, Leininger, Allen, Peplau, Henderson, Orlando, Rogers, Parse, Watson y Johnson. 
- Crear una teorización con el hilo conductor de Orem y las aportaciones de las autoras Roy, Leininger, Allen y Peplau, para aproximarnos a la práctica.

\section{Método:}

Instrumentos: los fundamentos teóricos enfermeros descritos por las diferentes autoras.

Procedimiento: Pensamiento crítico- reflexivo utilizando una estructura de mayor a menor nivel de abstracción y a través de la generación de ideas.

\section{Resultados:}

Un metaparadigma y teorización innovadores y actuales que tienen como base la teoría de Orem (teoría de alto rango), que guió, dio sentido, forma y fiabilidad a nuestro nuevo constructo.

\section{Conclusiones:}

Se observa que es posible la sinergia de las teorías y modelos de Enfermería así como otorgarles un enfoque actual y moderno. Se abren líneas futuras de investigación que fundamenten el autocuidado y el cuidado como objeto propio de nuestra disciplina.

\section{Palabras Clave:}

MeSH: Teoría; Enfermería; Orem; Roy; Liderazgo.

\section{ABSTRACT}

Title: Metaparadigm and innovative theorization on nursing theories and models.

\section{Objectives:}

- To develop a new metaparadigm as a compendium of the essential ideas and the most current theories and models written by Orem, Roy, Leininger, Allen, Peplau, Henderson, Orlando, Rogers, Parse, Watson and Johnson. 
- To develop a theorization following the leitmotif of Orem's theory and the contributions of authors such as Roy, Leininger, Allen and Peplau, to approach practice.

\title{
Method:
}

- Tools: Foundations described in the different theories we previously mentioned.

- Procedure: Critical-thoughtful thinking using a structure with a wide level of abstraction and generation of ideas.

\section{Results:}

An innovative metaparadigm following the base of Orem's theory (high ranking theory), which has led and supported our new development.

\section{Conclusions:}

We find out that it is possible to find synergies between theories and practical models in the Nursing Sector. New future researches focused on selfcare are open as a new discipline.

\section{Key words:}

MeSH: Nursing; Theory; Orem; Roy; Leadership.

\section{INTRODUCCIÓN}

\author{
"Todas las teorías son legítimas y ninguna tiene \\ importancia. Lo que importa es lo que se hace con ellas".
}

Jorge Luis Borges (1899-1986)

El presente estudio se ha desarrollado durante el curso académico 2011-12 del Máster Oficial en Ciencias de la Enfermería (Huelva). La idea surge tras el arduo trabajo de investigación en la asignatura de Teorías y Modelos de Enfermería y como resultado nace este nuevo constructo innovador y más actual, basado en los fundamentos teóricos de nuestra disciplina.

Nuestros objetivos son crear tanto un metaparadigma compendio de las ideas esenciales de todas las teóricas como una teorización con el hilo conductor de Orem y las aportaciones de las autoras Roy, Leininger, Allen y 
Peplau con el único fin de actualizar y acercar más a la práctica los modelos y teorías de Enfermería desfasados hoy día en el tiempo.

Tras una búsqueda bibliográfica realizada en las bases de datos Pubmed, Cuiden y Scielo los hallazgos resultantes únicamente son casos prácticos fundamentados con las teorías y modelos de Enfermería1, 2, 3; no obstante, no hemos encontrado ningún artículo que evidencie ni la repercusión actual de las teorías ni su complementariedad.

Introducimos este estudio con la siguiente reflexión: "la Enfermería tiene un rol educativo, una fuerza de maduración que intenta favorecer el avance de la personalidad en dirección de una vida personal y comunitaria creativa, constructiva y productiva" ${ }^{4}$.

\section{MÉTODO}

\section{Instrumentos:}

Para la elaboración del metaparadigma y de la teorización hemos utilizado los fundamentos teóricos enfermeros descritos por las diferentes autoras.

En el caso del metaparadigma utilizamos las ideas desarrollas por Orem, Henderson, Peplau, Leininger, Orlando, Parse, Watson, Johnson, Rogers, Roy, Allen mientras que para la elaboración de la teorización nos basamos en Orem, Roy, Leininger, Allen y Peplau.

Tras un estudio crítico-reflexivo sobre las teorías y modelos, surgió la idea de crear un nuevo constructo teórico más actual, así como un metaparadigma en el cual estuviesen integrados los conceptos e ideas de cada autora más afines a la realidad enfermera tal como se concibe hoy día.

\section{Procedimientos:}

La metodología seguida se desarrolla de mayor a menor nivel de abstracción. 
Para la elaboración del metaparadigma, partimos de los conceptos persona, entorno, salud y enfermería descritos por Orem y fuimos agregándole las ideas esenciales del metaparadigma de cada autora dando como resultado un compendio integra el enfoque de todas ellas.

Los criterios de inclusión fueron la selección de conceptos e ideas actuales desarrolladas en la práctica clínica y entendibles tal como se perciben hoy día por los profesionales enfermeros.

Los criterios de exclusión fueron desechar aquellas definiciones desfasadas en el tiempo, sin contemplar la interculturalidad e interdisciplinaridad que caracterizan a nuestra profesión.

De modo similar elaboramos un nuevo constructo teórico enfermero, innovador, actual, y realizable en la labor enfermera desarrollada.

Para ello partimos de la teoría de Dorothea Orem como hilo conductor; el motivo no es sino otro que es una teoría de alto rango ${ }^{5}$. Dicha teoría descrita por Orem está basada en la evidencia científica, en hechos reales, probados, y por tanto consideramos más que una razón de peso para que guiará y diera sentido, forma y fiabilidad a nuestro constructo, que surgió como base de una generación de ideas propuestas con el único fin de actualizar y acercar más a la práctica los modelos y teorías enfermeros desfasados actualmente en el tiempo.

Así pues, con Orem como cimiento construimos una teorización con las aportaciones de Roy, Leininger, Peplau y Allen aquellas autoras de las que pudimos exprimir y sacar ideas actuales, integrándolas desde otra perspectiva.

\section{RESULTADOS}

\section{Metaparadigma de Enfermería}

El metaparadigma representa la estructura más abstracta y global de nuestra disciplina ${ }^{5,6}$. Integra las definiciones conceptuales de persona, entorno, salud y Enfermería ${ }^{6}$ dotando así a nuestra profesión de un significado concreto y exclusivo. 


\section{Persona}

Ser humano con funciones biológicas, sociales y simbólicas; con potencial para aprender y desarrollarse ${ }^{7}$. Sujeto a las fuerzas de la naturaleza es capaz de autoconocerse, interpretar experiencias y llevar a cabo acciones beneficiosas. Inserta en un todo capaz de consecución de metas ${ }^{7}$ y con adaptación $^{8}$ que puede expresar verbal y no verbalmente ${ }^{9}$.

La suma de estas afirmaciones dan significado a un ser creativo ${ }^{10}$ visto como un campo de energía heliocéntrico (bio-psico-socio-espiritual) en desarrollo exponencial caracterizado por un equilibrio dinámico ${ }^{11}$ según su estado enérgico ${ }^{11}$ y en continuo contacto con su entorno- familia donde, en ocasiones, la enfermera establece una relación terapéutica ${ }^{12}$.

Es a su vez un ser unitario dentro de sus costumbres, cultura ${ }^{13,} 14$ espiritualidad y religión ${ }^{15}$ que busca conseguir una armonía total ${ }^{16}$ y es consciente de la esencia de su ser.

\section{Entorno}

El entorno queda definido como los campos de energía que constituyen la persona y el entorno que son inseparables y se rigen por una serie de principios en el que el de helicidad explica la tendencia a la diversificación creciente y creativa con ritmos incesantes de renovación ${ }^{17}{ }^{18}$.

Se considera el entorno como un ambiente cambiante con factores positivos y negativos, relevantes 0 insignificantes ${ }^{19}$ donde las personas cercanas interactúan con la persona ${ }^{12}$ que posee su propia concepción del mundo ${ }^{11}$, tanto físico como espiritual ${ }^{14}$.

Es el contexto donde se realizan los autocuidados ${ }^{7}$ y se aprenden los cuidados de salud con la familia o personas cercanas ${ }^{20}$, así como los cuidados que realizan las enfermeras ${ }^{21}$. Se tiende a la adaptación y regulación constante ${ }^{16}$.

\section{Salud}

Sentimiento de bienestar ${ }^{21}$, comodidad física y mental ${ }^{21}$ donde se alcanza 
un equilibrio, un nivel máximo de adaptación ${ }^{7}$ al entorno diferenciándola de las alteraciones y enfermedades importantes.

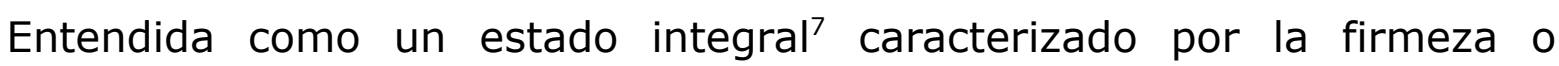
totalidad de la estructura y el funcionamiento corporal ${ }^{7}$, que implica un movimiento dirigido de personalidad y otros procesos propios del hombre hacia una vida creativa, constructiva y productiva tanto personal como en comunidad $^{12}$.

Proceso de actualización ${ }^{18}$ y percepción que no se puede cuantificar ni cualificar, surge de la interacción entorno-persona ${ }^{18}$.

Consta de aspectos: físicos, psicológicos, interpersonales, sociales y culturales.

Fuerza, voluntad y conocimiento han de dirigirse hacia la promoción del mantenimiento de la salud ${ }^{14}$ a través del autocuidado, del tratamiento y de la prevención de las enfermedades, donde la enfermería desarrolla su potencial para el óptimo funcionamiento del cuerpo humano.

\section{Enfermería}

Disciplina científica y humanística ${ }^{13}$ del arte $^{10}$ de cuidar. Nace del esfuerzo creativo de un ser humano por ayudar a otro u otros seres humanos. Se establece una relación terapéutica interpersonal ${ }^{22}$ con un perfil profesional específico ejerciendo roles, así mismo, dentro de un equipo transdisciplinar.

La enfermera adquiere el rol de compañera ${ }^{23}$, maestra ${ }^{22}$, sustituta ${ }^{22}$, asesora ${ }^{22}$, líder ${ }^{22}$ e investigadora. Se caracteriza por habilidades ${ }^{20,} 22$ en la autonomía ${ }^{11}$, el dinamismo, la colaboración ${ }^{20}$, la negociación ${ }^{20}$ y la coordinación ${ }^{20}$.

Actúa como un agente:

- promotor de la salud $^{10,20,23}$, el bienestar ${ }^{9,13}$ y la calidad de vida ${ }^{18}$ de las personas, familias y comunidades;

- $\quad$ protector de la dignidad humana ${ }^{18}$;

- de empatía 23 , consuelo y alivio frente sufrimiento; 
- de ayuda en el afrontamiento a la enfermedad y a la discapacidad ${ }^{13}$;

- favorecedor de una muerte tranquila ${ }^{13,23}$.

La disciplina de Enfermería englobará al individuo o al grupo en un entorno de continuos cambios, de constantes interacciones entre seres humanos influidos por una cultura ${ }^{13}$, unas creencias $y$ unos valores determinados.

\section{Teorización}

Las teorías tienen como objeto delimitar y especificar uno o varios conceptos sobre los cuáles se cimiente una ciencia5,6. Gracias a su nivel de abstracción y complejidad inferior con respecto al metaparadigma, actúan como fundamentos de la práctica asistencial de Enfermería.

En primer lugar, reforzamos los factores condicionantes de autocuidado descritos por Orem (autoconcepto, madurez, metas, cultura, conocimientos, familia y amigos) ${ }^{24}$ con el enfoque de Roy "persona como sistema holístico y adaptable" 25 .

Por consiguiente, el autocuidado ${ }^{26}$ sería el fruto de una respuesta adaptativa y derivada de un proceso cambiante donde los factores condicionantes son los estímulos ${ }^{25}$, mecanismos de afrontamientos ${ }^{25}$ y modos de adaptación de los individuos ${ }^{25}$.

Los estímulos se entienden como percepciones y pueden pertenecer a tres categorías:

- Focales. Internos o externos. Afectan inmediatamente al ser humano.

- Contextuales. "Otros estímulos presentes en la situación que contribuyen a originar el estimulo focal".

- Residuales. Factores del entorno de dentro o fuera del ser humano que provocan unos efectos no claramente definidos en la situación del momento.

De la influencia que ejercen dichos estímulos sobre las personas se originan mecanismos de afrontamiento innatos o adquiridos. Se identifican dos 
subsistemas:

- Mecanismo Regulador. Constituido por el sistema neuronal químico y endocrino.

- Mecanismo Relacionador. Compuesto por cuatro canales que intervienen en el afrontamiento: perceptivo, informativo, juicio personal y emociones.

Estos mecanismos generarán uno o varios modos de adaptación en los individuos:

- Función fisiológica. Corresponde a los requisitos universales descritos por Orem.

- Función del rol. Hace referencia a la función que desempeña la persona dentro de la sociedad.

- Autoconcepto. Centrado en los aspectos psicológicos y espirituales del individuo.

- Interdependencia. Determinado por las relaciones interpersonales.

La respuesta resultante es el autocuidado. No obstante, si todo este proceso no se desarrolla con normalidad surgiría una respuesta individual desadaptativa conocida como situación de déficit de autocuidado.

La cultura ${ }^{13,14}$ constituye un factor condicionante transversal afectando tanto al proceso como al resultado.

Diferenciamos tres niveles de adaptación: integrado, comprometido y compensatorio.

\section{i) Nivel de Adaptación Integrado}

Respuesta eficaz de la persona ${ }^{25}$. Existe equilibrio entre sus requisitos de autocuidado y su capacidad de autocuidarse ${ }^{24}$. La enfermera actúa como agente promotor de la salud ${ }^{20}$ mediante actuaciones desempeñadas en el ámbito de atención primaria y con el objetivo de conservar dicho equilibrio. 


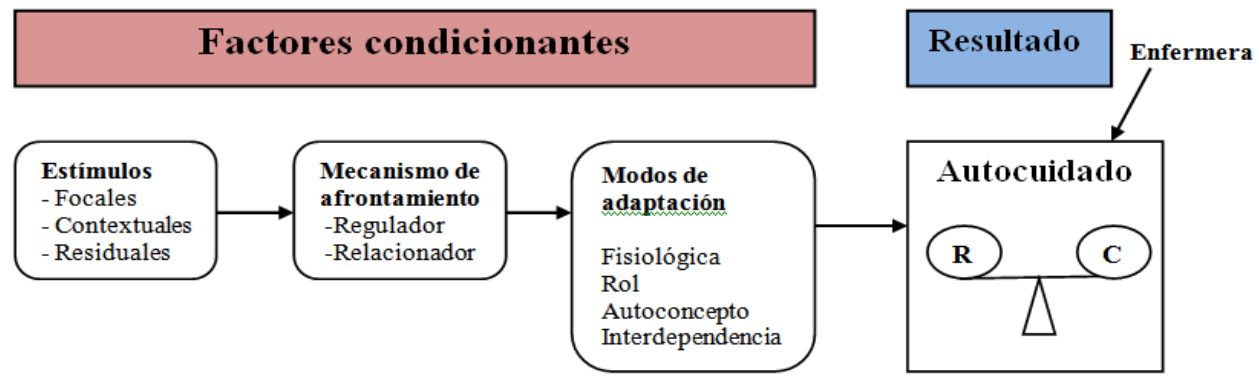

Factor condicionante transversal. Cultura

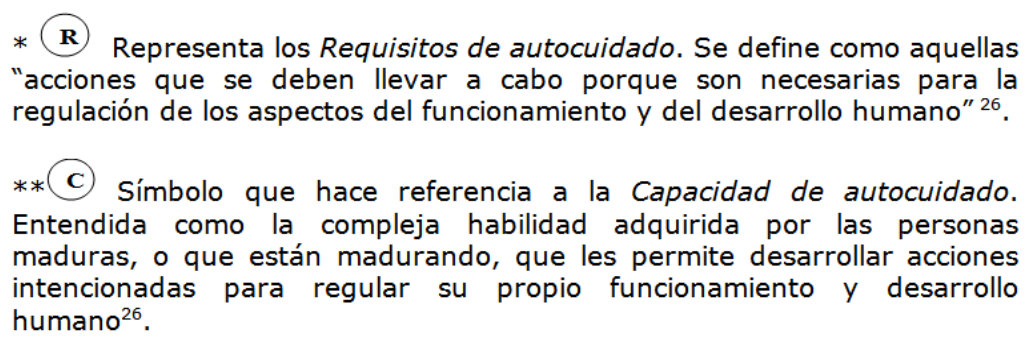

\section{ii) Nivel de Adaptación Comprometida.}

Respuesta ineficaz. Los requisitos de autocuidado superan a las capacidades de la persona para autocuidarse ${ }^{25}$.

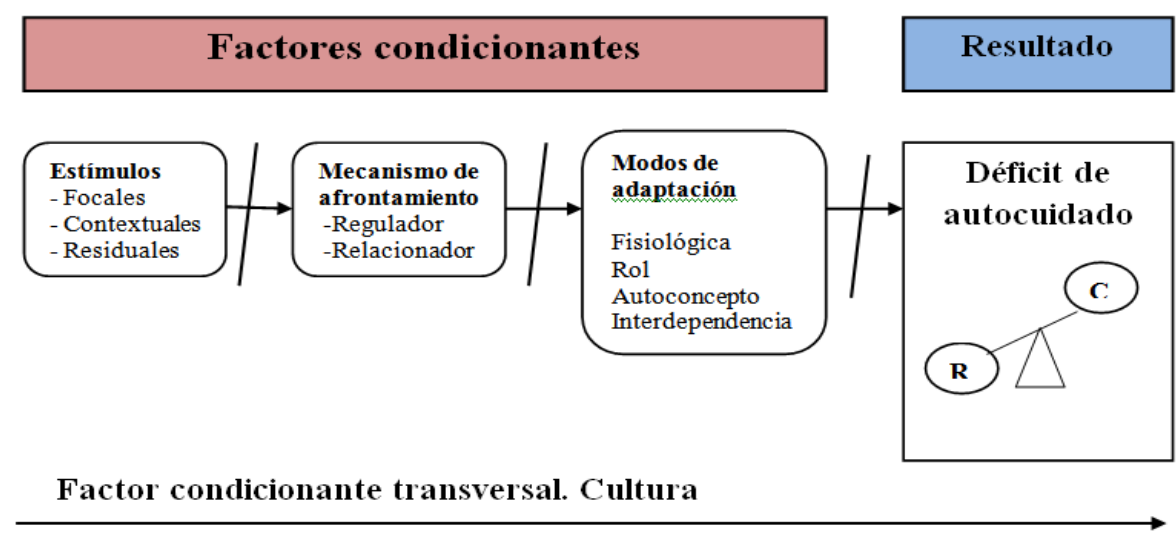

El resultado del proceso de adaptación no es satisfactorio surgiendo requisitos de desviación de la salud, es decir, aquellos que aparecen en las personas afectadas de un problema de salud ${ }^{26}$. Requieren de:

- Buscar y asegurar asistencia sanitaria.

- Observar y ser consciente de la enfermedad en sí misma.

La Teoría del Déficit de Autocuidado establece que las personas con limitaciones en su autocuidado o limitaciones en el cuidado prestado a otros individuos precisan de profesionales de Enfermería.

Dichas personas se encuentran en situación de crisis y se caracterizan por: 
- vivirlo de forma aguda, estrictamente personal y como una situación de ansiedad;

- la necesidad de expresar sus sentimientos;

- la necesidad de un profesional que le ayude a hacer balance de su situación.

La búsqueda de la adaptación se concreta en las siguientes etapas ${ }^{12}$.

Primera fase. Orientación.

Se manifiesta con: agitación y actividad; emociones y sentimientos caóticos; pocas posibilidades de reflexión.

Segunda fase. Identificación.

Identificada con: una visión oscura de las cosas; una gran tensión interior; una ausencia de recursos; la necesidad de canalizar la angustia.

\section{iii) Nivel de Adaptación Compensatoria}

Transformación de una respuesta ineficaz en adaptativa gracias a la intervención por parte profesional de Enfermería y/o del acompañante. La persona retorna a su situación de equilibrio entre requisitos y capacidades de autocuidarse.

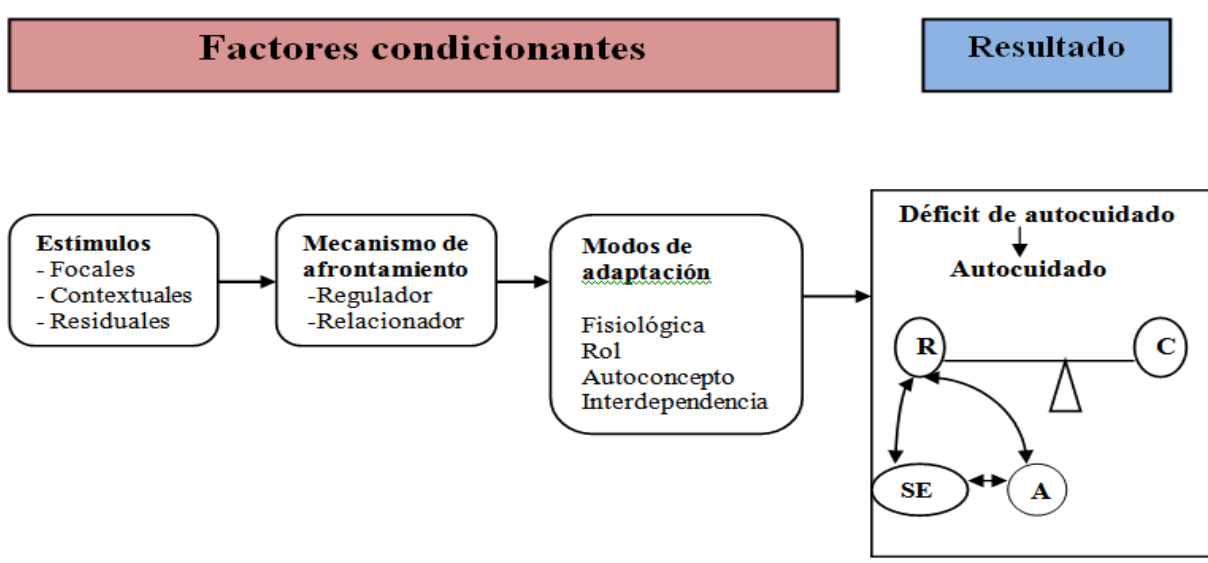

Factor condicionante transversal. Cultura

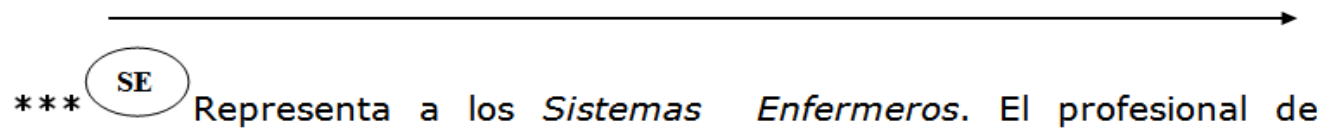
Enfermería actúa como agente de cuidado terapéutico y establecerá una relación interpersonal no solo con la persona cuidada sino también con su acompañante $^{23}$. Encuadrándose aquí Teoría de los Sistemas Enfermeros ${ }^{28}$.

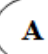

Simboliza al Acompañante, ententido como un agente de cuidado dependiente. 
En este nivel surgen los siguientes requisitos de desviación de la salud en las personas cuidadas:

- cumplir las prescripciones de cuidados y observar sus efectos;

- aprender a vivir con los efectos derivados de los problemas de salud o del tratamiento.

Y se concretan en las siguientes etapas adaptativas ${ }^{12}$ :

Tercera fase. Profundización.

Determinada por: la posibilidad de obtener recursos y de intervenir; redefinir el problema y buscar alternativas.

\section{> Cuarta fase. Resolución.}

Concluye la relación terapéutica.

El profesional de Enfermería desarrolla funciones en cuatro dimensiones -asistencia, docencia, gestión e investigación ${ }^{25}$ - que conllevan a la adquisición de diferentes roles: persona con recursos ${ }^{22}$, asesor ${ }^{22}$, maestro ${ }^{22}$, sustituto ${ }^{22}, 26$ y compañero ${ }^{23}$; y todas ellos se agrupan en el rol líder de los cuidados.

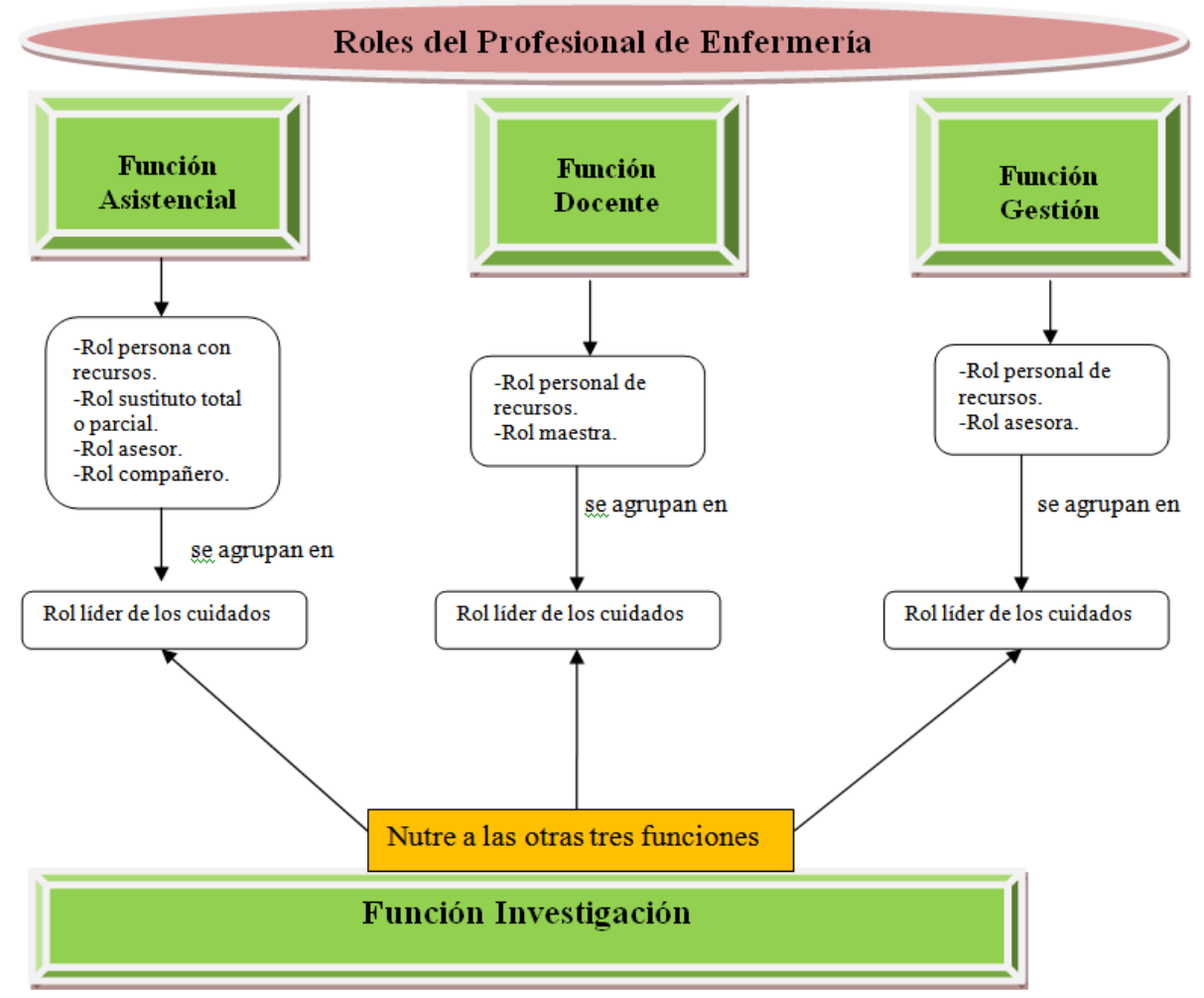


La enfermera dispone de los recursos materiales y de las habilidades en la comunicación que le permitirán establecer una relación terapéutica efectiva.

Rol Asesor 22

El profesional de Enfermería informa a la persona cuidada sobre su estado de salud y orienta en los autocuidados y posibles alternativas.

Rol Maestro 22

- Categoría instructivo: el profesional proporciona información.

- Categoría experiencia: cuando la persona utiliza su experiencia para crear otras nuevas.

Rol Sustituto 22,26

La persona cuidada se proyecta en el profesional de Enfermería el cuál delimita las áreas de dependencia, interdependencia e independencia.

Rol Compañero ${ }^{23}$

El profesional mantiene una actitud cálida y es capaz de empatizar con la persona.

Rol Líder de los cuidados 22

Engloba a todos los roles anteriormente mencionados ya que:

- El profesional de Enfermería asistencial partiendo de los requisitos y capacidades de la persona cuidada actuará como líder promotor de su autocuidado. Asimismo, valorará la actitud del agente de cuidado dependiente como invasivo, colaborador o pasivo, con el fin de determinar su intervención.

- El profesional de Enfermería docente liderará la enseñanza sobre los cuidados.

- El profesional de Enfermería gestor se encargará de liderar los recursos humanos y administrar los recursos materiales.

Concibiéndose al líder como "una persona capaz de ejercer influencia en 
otros, dirigirlos y guiarlos efectivamente hacia el logro de objetivos y metas organizacionales y personales" 28.

Además de los roles adquiridos, los profesionales de Enfermería emplean diferentes herramientas que aseguren la eficacia de la relación de ayuda. Destacando el contrato como instrumento de compromiso del individuo hacia su autocuidado.

Para finalizar, las prescripciones de cuidados junto con los acuerdos verbales establecidos entre persona cuidada- profesional de Enfermería actualmente son modalidades de dichos contratos.

\section{DISCUSIÓN}

Tras el remodelado y construcción de nuevos fundamentos teóricos a partir de las potencialidades propias de cada autora, hemos logrado no solo demostrar que es posible la sinergia de las teorías y modelos de Enfermería, sino además otorgarles un enfoque actual y moderno.

Destacar como factor limitante de nuestro teorización la inminente necesidad de realizar investigaciones que refuercen la complementariedad teórica. Así como, serie de casos que correlacionen la práctica con la teoría.

Consideramos que nuestra profesión debe dar una respuesta coherente y acorde a las necesidades de la población actual. Nuestro estudio da lugar a futuras líneas de investigación que sustenten el autocuidado y el cuidado como objeto propio de nuestra disciplina $29,30$.

La Practica Basada en la Evidencia y en unos fundamentos teóricoscientíficos constituye el árbol de nuestro ser, saber y quehacer profesional ${ }^{29}$. Un árbol que delimita nuestros valores, pensamientos y comportamientos. $Y$ por supuesto, un árbol cuyo crecimiento solo depende de nosotros mismos. 


\section{BIBLIOGRAFÍA}

1) José León, W., Mendoza, M. J. Aplicación del Modelo Teórico de Dorothea Orem en un caso de Insuficiencia Renal Crónica. Paraninfo Digital. 2011. Disponible en: <<http://www.index-f.com/para/n11-12/188d.php>>

2) Navarro, Y., Castro, M. Modelo de Dorothea Orem aplicado a un grupo comunitario a través del proceso de enfermería. Enfermería Global. 2010. Vol 19.

3) Molina, A. S., Suazo, S.V. Teoria de Orem aplicada a intervenciones durante embarazo y climaterio. Rev Bras Enferm, Brasília .2009; Vol 62; No4. P. 613-9.

4) Freese, B. T. Betty Neuman. Modelo de Sistemas. En: Marriner A, Raile, M. Modelos y teorías en enfermería. 6a ed. Barcelona: Elsevier; 2007. p. 329.

5) Sarabia, A., Rojas, L., Meléndez, R.M. Teoría: alcances y niveles para explicar la práctica de Enfermería. Biblioteca Lascasas. 2011; Vol 7, No 3. Disponible en: $\quad<$ http: //www.indexf.com/lascasas/documentos/lc0622.php >>

6) Kuerten, P., Lenise, M. Modelo de cuidado. ¿Qué es y cómo elaborarlo? Índex de Enfermería. 2008; Vol 17, No 2.

7) Kérouac, S., Pepin, J., Ducharme, F., Duquette, A., Major, F. Concepciones de Nightingale a Parse. En: El pensamiento enfermero. $4^{\mathrm{a}}$ ed. Barcelona: Masson; 2005. p. 29-31.

8) Kérouac, S., Pepin, J., Ducharme, F., Duquette, A., Major, F. Concepciones de Nightingale a Parse. En: El pensamiento enfermero. $4^{a}$ ed. Barcelona: Masson; 2005. p. 34-6.

9) Jean, N. Ida Jean Orlando. Teoría del proceso de enfermería. En: Marriner A, Raile, M. Modelos y teorías en enfermería. 6a ed. Barcelona: Elsevier; 2007. p. 431-51.

10) Gunther, M.E. Martha E. Rogers. Seres humanos unitarios. En: Marriner A, Raile M. Modelos y teorías en enfermería. $6^{a}$ ed. Barcelona: Elsevier; 2007. p. 244- 66.

11) Kérouac, S., Pepin, J., Ducharme, F., Duquette, A., Major, F. 
Concepciones de Nightingale a Parse. En: El pensamiento enfermero. $4^{\mathrm{a}}$ ed. Barcelona: Masson; 2005. p.53.

12) Kérouac, S., Pepin, J., Ducharme, F., Duquette, A., Major, F. Concepciones de Nightingale a Parse. En: El pensamiento enfermero. $4^{a}$ ed. Barcelona: Masson; 2005. p. 32-4; 49.

13) McFarland, M. Madeleine Leininger. Teoría de la diversidad y de la universalidad de los cuidados culturales. En: Marriner A, Raile M. Modelos y teorías en enfermería. 6a ed. Barcelona: Elsevier; 2007. p.472- 98.

14) Kérouac, S., Pepin, J., Ducharme, F., Duquette, A., Major, F. Concepciones de Nightingale a Parse. En: El pensamiento enfermero. $4^{a}$ ed. Barcelona: Masson; 2005. p. 45-7.

15) 15 Kérouac, S., Pepin, J., Ducharme, F., Duquette, A., Major, F. Concepciones de Nightingale a Parse. En: El pensamiento enfermero. $4^{\mathrm{a}}$ ed. Barcelona: Masson; 2005. p. 28-9.

16) Kérouac, S., Pepin, J., Ducharme, F., Duquette, A., Major, F. Concepciones de Nightingale a Parse. En: El pensamiento enfermero. $4^{a}$ ed. Barcelona: Masson; 2005. p. 43-5.

17) Kérouac, S., Pepin, J., Ducharme, F., Duquette, A., Major, F. Concepciones de Nightingale a Parse. En: El pensamiento enfermero. $4^{a}$ ed. Barcelona: Masson; 2005. p. 39-40.

18) Kérouac, S., Pepin, J., Ducharme, F., Duquette, A., Major, F. Concepciones de Nightingale a Parse. En: El pensamiento enfermero. $4^{a}$ ed. Barcelona: Masson; 2005. p. 41-2.

19) Philips K.D. Sor Callista Roy: Modelo de adaptación. En: Marriner A. y Raile M. Modelos y Teorías en enfermería. Barcelona: Elsevier; 2007.p.361.

20) Kérouac, S., Pepin, J., Ducharme, F., Duquette, A., Major, F. Concepciones de Nightingale a Parse. En: El pensamiento enfermero. $4^{a}$ ed. Barcelona: Masson; 2005. p. 36-8.

21) Kérouac, S., Pepin, J., Ducharme, F., Duquette, A., Major, F. Concepciones de Nightingale a Parse. En: El pensamiento enfermero. $4^{a}$ ed. Barcelona: Masson; 2005. p. 50.

Marriner, A. Teorías de la enfermería de importancia histórica. En: 
Marriner A, Raile M. Modelos y teorías en enfermería. 6a ed. Barcelona: Elsevier; 2007. p.54.

23) Marriner, A. Teorías de la enfermería de importancia histórica. En: Marriner A, Raile M. Modelos y teorías en enfermería. 6a ed. Barcelona: Elsevier; 2007. p. 54-6.

24) Fernández, C. Autocuidados, requisitos de autocuidado, demanda de autocuidado terapéutico. En: Modelo de Orem. Conceptos de Enfermería en la práctica. $2^{a}$ ed. Barcelona: Masson. p.134.

25) Phillips, K.D. Callista Roy. Modelo de adaptación. En: Marriner A, Raile, M. Modelos y teorías en enfermería. 6a ed. Barcelona: Elsevier; 2007. p. 353-84.

26) Taylor, S. G. Dorothea E. Orem. Teoría del deficit de autocuidado. En: Marriner A, Raile M. Modelos y teorías en enfermería. 6a ed. Barcelona: Elsevier; 2007. p. 267-95.

27) Libro Blanco de la ANECA para Enfermería. Agencia Nacional de Evaluación de la Calidad y la Acreditación, 2005. Disponible en: <<http://www.aneca.es/var/media/150360/libroblanco_jun05_enfermeri a.pdf $>>$

28) Guevara, R.M., Baltazar, R.M. Liderazgo de Enfermería. Desarrollo Cientif Enferm. 2010; Vol. 18; No 6. p. 265-68.

29) Eugenia, V., Guarnizo, M. Enfermería como disciplina. Revista Colombiana de Enfermería. 2011; Vol 6, No 6. p. 73-81.

30) De la Cuesta, C. Investigación cualitativa y el desarrollo del conocimiento en Enfermería. Texto Contexto Enferm, Florianópolis. 2010; Vol 19, No 4. p. 762-6. 